\title{
Cálculo de criticalidade pela teoria da difusão de nêutrons: uma análise comparativa de aproximações da densidade de corrente
}

\author{
Criticality calculations by neutron diffusion theory: \\ a comparative analysis of current density approximations
}

Rodrigo Zanette

Universidade Federal do Rio Grande do Sul (UFRGS), Programa de Pós Graduação em

Matemática Aplicada, Porto Alegre, RS, Brasil

https://orcid.org/0000-0002-5183-3438, rodrigozanette@hotmail.com

Liliane Basso Barichello

Universidade Federal do Rio Grande do Sul (UFRGS), Instituto de Matemática e Estatística, Porto

Alegre, RS, Brasil

https://orcid.org/0000-0003-2519-086X, Ibaric@mat.ufrgs.br

Claudio Zen Petersen

Universidade Federal de Pelotas (UFPel), Instituto de Física e Matemática, Pelotas, RS, Brasil

https://orcid.org/0000-0002-4720-6888, claudio.petersen@ufpel.edu.br

Informações do Artigo

\section{Como citar este artigo}

ZANETTE, Rodrigo;

BARICHELLO, Liliane Basso;

PETERSEN, Claudio Zen. Cálculo

de criticalidade pela teoria de difusão de nêutrons: uma análise comparativa de aproximação da densidade de corrente. REMAT:

\section{Revista Eletrônica da}

Matemática, Bento Gonçalves,

RS, v. 6, n. 2, p. e4006, nov. 2020.

DOI: https://doi.org/10.35819/ remat2020v6i2id4248

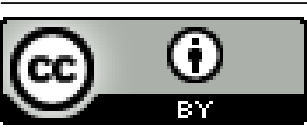

Histórico do Artigo

Submissão: 08 de junho de 2020.

Aceite: 21 de julho de 2020 .

Palavras-chave

Equação de Difusão de Nêutrons

Criticalidade

Integração Nodal

Densidade de Corrente de Nêutrons

\section{Resumo}

Este trabalho apresenta uma análise comparativa entre algumas formas de aproximação das densidades de corrente em cálculos de criticalidade usando a teoria da difusão de nêutrons. Como os reatores nucleares são compostos por diversos materiais, definindo regiões heterogêneas onde os parâmetros nucleares variam de forma significativa, é fundamental que as correntes sejam expressas de tal forma a preservar continuidade nas interfaces das regiões. A partir de uma integração nodal na equação da difusão de nêutrons estacionária, apresentamos quatro propostas de aproximação para as densidades de corrente nas interfaces. Uma vez construído o modelo, o cálculo do parâmetro que define a criticalidade depende da determinação do autovalor dominante. Aqui apresentamos e discutimos três métodos do cálculo deste autovalor. A comparação dos resultados numéricos é realizada a partir de três problemas teste, em meios heterogêneos, disponíveis na literatura. Os resultados obtidos indicam que as aproximações mais efetivas para as densidades de corrente nas interfaces, para cálculos do autovalor e dos fluxos, são aquelas que relacionam os coeficientes de difusão dos dois nós comuns à interface (propostas 3 e 4). Além disso, o método da secante se mostrou mais eficiente para determinação do parâmetro da criticalidade. 


\section{Keywords}

Neutron Diffusion Equation

Criticality

Nodal Integration

Neutron Current Density

\begin{abstract}
This work presents a comparative analysis of different approaches to approximate current densities in criticality calculations using the neutron diffusion theory. As the nuclear reactors are composed of several materials, defining heterogeneous regions, where the nuclear parameters vary significantly, it is necessary to express the currents in such a way as to preserve continuity at the interfaces of the regions. Based on a nodal integration applied to the stationary neutron diffusion equation, we present four proposals to approximate the current densities at the interfaces. Once the model is built, the calculation of the parameter that defines criticality depends on the determination of the dominant eigenvalue. Here we present and discuss three methods of calculating this eigenvalue. The comparison of the numerical results is carried out on the basis of three test problems, in heterogeneous media, available in the literature. The results obtained indicate that the most effective approximations for the current densities at the interfaces, for calculating the eigenvalue and the fluxes, are those that combine the diffusion coefficients of the two common nodes to the interface (proposals 3 and 4). In addition, the secant method proved to be more efficient in determining the criticality parameter.
\end{abstract}

\section{Introdução}

A solução do problema da criticalidade nos reatores nucleares é de fundamental importância para o licenciamento das usinas nucleares. A criticalidade está associada ao comportamento evolutivo do número de nêutrons ao longo de sucessivas gerações de fissão nuclear. A fissão nuclear ocorre quando o núcleo de um átomo de urânio, por exemplo U-235, absorve um nêutron, ficando assim instável a tal ponto de romper-se (fissionar-se). Além de gerar dois produtos de fissão com emissão, em média, de 2 a 3 nêutrons em cada evento de fissão (LEWIS, 2008), esses novos nêutrons podem causar novas fissões gerando, assim, uma reação em cadeia. Além de causarem novas fissões, alguns desses novos nêutrons podem ser absorvidos por materiais que compõem o núcleo do reator, tais como: moderador, refrigerante, elementos de controle. Os demais nêutrons podem sofrer captura radioativa pelo U-238, nas regiões de ressonâncias do combustível e, segundo Duderstadt e Hamilton (1976), apenas alguns nêutrons conseguem escapar dessas regiões. Cabe ressaltar que, desde a fissão rápida até a absorção térmica de nêutrons no combustível, podem ocorrer fugas rápidas e térmicas de nêutrons no reator. O ideal é que a relação em perdas e ganhos de nêutrons se mantenha em equilíbrio, ou seja, que o reator funcione em estado crítico.

Nesse contexto, a teoria da difusão de nêutrons é um modelo matemático que descreve, de forma satisfatória, o comportamento neutrônico dos reatores nucleares (DUDERSTADT; HAMILTON, 1976, STACEY, 2001). A equação da difusão de nêutrons estacionária é utilizada para prever o 
comportamento da população de nêutrons a partir da composição geométrica e material do reator. Esta equação é adaptada para um problema de autovalor $K$, pois a quantidade de material nuclear deve ser equilibrada com a quantidade de materiais absorvedores que compõem o núcleo, a fim de manter a distribuição de fluxo estacionário, que é a condição ideal de operação do reator. Este autovalor representa a razão entre o número de nêutrons de duas sucessivas gerações de fissão, de tal forma que se deseja obter o valor de $K$ próximo da unidade. Caso contrário, precisa-se escolher uma nova combinação entre geometria e composição, para que se atinja a criticalidade desejada. Do ponto de vista do modelo matemático, a determinação desse parâmetro se refere à solução de um problema de autovalor, no qual apenas o autovalor dominante $K$ corresponde aos autovetores reais e não-negativos em todos os pontos do sistema e, portanto, fisicamente relevante (LAMARSH; BARATTA, 2017). Nos problemas realistas em física de reatores, a dependência dos parâmetros do modelo com relação à variável energia é um ponto relevante a ser observado. O espectro de energia é muito amplo, o que torna comum a utilização do chamado modelo multigrupo, no qual os nêutrons são divididos em grupos, de acordo com sua faixa de energia cinética. Assim, em cada grupo, assume-se que a energia do nêutron seja constante (DUDERSTADT; HAMILTON, 1976).

Além das condições de contorno, faz-se necessário, para a solução desse tipo de problema, especificar as condições de interfaces entre os diferentes meios presentes no interior do núcleo de um reator, tais como a interface entre o material combustível e o refletor. O fluxo e a corrente de nêutrons devem ser contínuos através das interfaces. Os eventos de fissão de nêutrons ocorrem nas regiões de combustíveis dos reatores nucleares, entretanto, seu cálculo global precisa considerar as outras regiões: moderador, refrigerante e núcleo estrutural; na qual os parâmetros nucleares variam de forma significativa. Essas mudanças dos parâmetros acarretam diversas consequências para os cálculos globais em física de reatores, como uma maior sensibilidade e perturbações do fluxo e da densidade de corrente perto das fronteiras entre as diferentes regiões. Dessa forma, faz-se necessário determiná-los com precisão nesses locais, atendendo as condições de continuidade.

Neste trabalho, investiga-se a influência de diferentes aproximações das densidades de correntes nas interfaces, em problemas de difusão multigrupo em geometria unidimensional. Além disso, visto a importância de se obter o valor da criticalidade dos reatores nucleares com precisão e agilidade, apresenta-se uma análise comparativa entre os métodos da bissecção, da secante e iterativo de fonte. Dessa forma, na Seção 2, introduzimos a formulação matemática do problema e as propostas de aproximação das correntes nas interfaces. Na Seção 3, apresentamos e discutimos os resultados numéricos. Por fim, na Seção 4, indicamos algumas conclusões. 


\section{Formulação matemática}

A equação da difusão de nêutrons multigrupo estacionária unidimensional em geometria cartesiana definida como um problema de autovalor é dado por (DUDERSTADT; HAMILTON, 1976)

$$
\frac{d}{d x}\left(-D_{g}(x) \frac{d}{d x} \phi_{g}(x)\right)+\Sigma_{R g}(x) \phi_{g}(x)=\frac{\chi_{g}}{K} \sum_{g^{\prime}=1}^{G} \nu_{g^{\prime}} \Sigma_{f g^{\prime}}(x) \phi_{g^{\prime}}(x)+\sum_{\substack{g^{\prime}=1 \\ g^{\prime} \neq g}}^{G} \Sigma_{s g^{\prime} g}(x) \phi_{g^{\prime}}(x),
$$

onde $x \in\left[x_{0}, x_{N}\right]$ e $g=1, \ldots, G$ são os grupos de energia. Para cada grupo de energia $g: D_{g}$ é o coeficiente de difusão, $[\mathrm{cm}] ; \phi_{g}(x)$ é o fluxo escalar de nêutrons, $\left[\mathrm{cm}^{-2} \mathrm{~s}^{-1}\right] ; \Sigma_{R g}$ é a seção de choque macroscópica de remoção, $\left[\mathrm{cm}^{-1}\right] ; \chi_{g}$ é o espectro integrado de fissão; $\nu_{g}$ é o número médio de nêutrons liberado por fissão; $\Sigma_{f g}$ é a seção de choque macroscópica de fissão, $\left[\mathrm{cm}^{-1}\right] ; \Sigma_{s g^{\prime} g}$ é a seção de choque macroscópica de espalhamento do grupo $g^{\prime}$ para $\circ g$, $\left[\mathrm{cm}^{-1}\right]$, e $K$ é o fator de multiplicação efetivo. As condições de contorno mais usuais para o sistema de equações (1) são do tipo de Dirichlet, Neumann ou Robin, escritas na forma

$$
\left.a_{g} \phi_{g}(x)\right|_{x=x_{b}}+\left.b_{g} \frac{d}{d x} \phi_{g}(x)\right|_{x=x_{b}}=0
$$

onde $\left|a_{g}\right|+\left|b_{g}\right|>0$ para $a_{g}$ e $b_{g}$ constantes reais e $x_{b}=x_{0}$ ou $x_{b}=x_{N}$.

Inicialmente consideramos a partição do domínio em $N$ nós, ou sub-domínios, de igual largura $\Delta x$, sendo que $x \in\left[x_{n-1}, x_{n}\right]$ no nó $n$. Seguindo então uma técnica de integração centrada na malha (GROSSMAN; HENNART, 2007), integramos a Eq. (1) para todo $x \in\left[x_{n-1}, x_{n}\right]$ e dividimos o resultado por $\Delta x$ para obtermos

$$
\frac{1}{\Delta x}\left(-\left.D_{g}^{(n)} \frac{d}{d x} \phi_{g}^{(n)}(x)\right|_{x=x_{n-1}} ^{x=x_{n}}\right)+\Sigma_{R g}^{(n)} \bar{\phi}_{g}^{(n)}=\frac{\chi_{g}}{K} \sum_{g^{\prime}=1}^{G} \nu_{g^{\prime}} \Sigma_{f g^{\prime}}^{(n)} \bar{\phi}_{g^{\prime}}^{(n)}+\sum_{\substack{g^{\prime}=1 \\ g^{\prime} \neq g}}^{G} \Sigma_{s g^{\prime} g}^{(n)} \bar{\phi}_{g^{\prime}}^{(n)}
$$

onde $\bar{\phi}_{g}^{(n)}$ é o fluxo escalar médio do grupo $g$ no nó $n$ definido por

$$
\bar{\phi}_{g}^{(n)}=\frac{1}{\Delta x} \int_{x_{n-1}}^{x_{n}} \phi_{g}^{(n)}(x) d x
$$

Além disso, definimos $J_{g}^{(n)}(x)$ como a densidade de corrente do grupo $g$ no nó $n$ 


$$
J_{g}^{(n)}(x)=-D_{g}^{(n)} \frac{d}{d x} \phi_{g}^{(n)}(x)
$$

Ao substituirmos a Eq. (5) na Eq. (3) obtemos um conjunto de equações em função dos fluxos médios e das correntes,

$$
\frac{1}{\Delta x}\left(J_{g}^{(n)}\left(x_{n}\right)-J_{g}^{(n)}\left(x_{n-1}\right)\right)+\Sigma_{R g}^{(n)} \bar{\phi}_{g}^{(n)}=\frac{\chi_{g}}{K} \sum_{g^{\prime}=1}^{G} \nu_{g^{\prime}} \Sigma_{f g^{\prime}}^{(n)} \bar{\phi}_{g^{\prime}}^{(n)}+\sum_{\substack{g^{\prime}=1 \\ g^{\prime} \neq g}}^{G} \Sigma_{s g^{\prime} g}^{(n)} \bar{\phi}_{g^{\prime}}^{(n)} .
$$

Note que temos $G N$ incógnitas relativas aos fluxos médios e $G(N+1)$ incógnitas relativas às densidades de corrente nos contornos do domínio e interfaces dos nós, porém, apenas $G N$ equações. Essa é uma questão usual em metodologias nodais. De forma que torna-se necessária a introdução de equações auxiliares para resolução do problema. Neste trabalho, propomos quatro formas de aproximação das densidades de corrente, em termos dos fluxos médios, de forma a reduzir o número de incógnitas do problema para $G N$ (fluxos médios), além do autovalor $K$.

- Proposta 1: Uma primeira proposta que sugerimos consiste em aproximar a derivada presente na expressão das densidades de corrente, Eq. (5), em termos de dois valores dos fluxos médios. Assim, a aproximação para a densidade de corrente, na interface direita do nó $n$, é escrita como

$$
J_{g}^{(n)}\left(x_{n}\right) \cong-D_{g}^{(n)}\left(\frac{\bar{\phi}_{g}^{(n+1)}-\bar{\phi}_{g}^{(n)}}{\Delta x}\right)
$$

e a aproximação, na interface esquerda do nó $n$, é escrita como

$$
J_{g}^{(n)}\left(x_{n-1}\right) \cong-D_{g}^{(n)}\left(\frac{\bar{\phi}_{g}^{(n)}-\bar{\phi}_{g}^{(n-1)}}{\Delta x}\right)
$$

Notamos, no entanto, que, neste caso, a condição de continuidade da densidade de corrente nas interfaces $x_{n-1}$,

$$
J_{g}^{(n)}\left(x_{n-1}\right)=J_{g}^{(n-1)}\left(x_{n-1}\right),
$$

não é atendida, quando $D_{g}^{(n)}$ é diferente de $D_{g}^{(n-1)}$. 
- Proposta 2: A segunda proposta que construímos é inspirada nas aproximações regressivas, na qual o coeficiente da difusão da aproximação da corrente no contorno é referente ao nó à sua esquerda. Assim, escrevemos a aproximação para a densidade de corrente na interface direita do nó $n$ como

$$
J_{g}^{(n)}\left(x_{n}\right) \cong-D_{g}^{(n)}\left(\frac{\bar{\phi}_{g}^{(n+1)}-\bar{\phi}_{g}^{(n)}}{\Delta x}\right)
$$

e a aproximação na interface esquerda do nó $n$ como

$$
J_{g}^{(n)}\left(x_{n-1}\right) \cong-D_{g}^{(n-1)}\left(\frac{\bar{\phi}_{g}^{(n)}-\bar{\phi}_{g}^{(n-1)}}{\Delta x}\right) .
$$

- Proposta 3: Essa proposta é desenvolvida a partir de aproximações que utilizam os fluxos médios de cada nó e dos fluxos nos contornos, conforme apresentado por Abreu (2017). Para o contorno à direita do nó $n$

$$
J_{g}^{(n)}\left(x_{n}\right) \cong-D_{g}^{(n)}\left(\frac{\phi_{g}\left(x_{n}\right)-\bar{\phi}_{g}^{(n)}}{\Delta x / 2}\right)
$$

e para o contorno à esquerda do nó $n$

$$
J_{g}^{(n)}\left(x_{n-1}\right) \cong-D_{g}^{(n)}\left(\frac{\bar{\phi}_{g}^{(n)}-\phi_{g}\left(x_{n-1}\right)}{\Delta x / 2}\right) .
$$

Nesse caso, quando impomos a condição de continuidade da densidade de corrente, Eq. (9), em $x_{n}$, obtemos

$$
\phi_{g}\left(x_{n}\right)=\frac{D_{g}^{(n+1)} \bar{\phi}_{g}^{(n+1)}+D_{g}^{(n)} \bar{\phi}_{g}^{(n)}}{D_{g}^{(n+1)}+D_{g}^{(n)}} .
$$

e quando impomos essa mesma condição de continuidade em $x_{n-1}$, obtemos

$$
\phi_{g}\left(x_{n-1}\right)=\frac{D_{g}^{(n)} \bar{\phi}_{g}^{(n)}+D_{g}^{(n-1)} \bar{\phi}_{g}^{(n-1)}}{D_{g}^{(n)}+D_{g}^{(n-1)}} .
$$

Por fim, substituindo a Eq. (14) na Eq. (12), obtemos uma aproximação da corrente na interface direita do nó $n$

$$
J_{g}^{(n)}\left(x_{n}\right) \cong-\frac{2 D_{g}^{(n+1)} D_{g}^{(n)}}{D_{g}^{(n+1)}+D_{g}^{(n)}}\left(\frac{\bar{\phi}_{g}^{(n+1)}-\bar{\phi}_{g}^{(n)}}{\Delta x}\right)
$$


e substituindo a Eq. (15) na Eq. (13), obtemos uma aproximação da corrente na interface esquerda do nó $n$

$$
J_{g}^{(n)}\left(x_{n-1}\right) \cong-\frac{2 D_{g}^{(n)} D_{g}^{(n-1)}}{D_{g}^{(n)}+D_{g}^{(n-1)}}\left(\frac{\bar{\phi}_{g}^{(n)}-\bar{\phi}_{g}^{(n-1)}}{\Delta x}\right)
$$

- Proposta 4: Esta proposta desenvolvemos a partir da média aritmética dos coeficientes da difusão e da aproximação da derivada por dois valores. Em ambos os casos, os valores dos coeficientes de difusão e dos fluxos médios são referentes aos nós vizinhos de cada interface, conforme podemos ver no esquema da Figura 1 para a interface à esquerda do nó $n$.

Figura 1 - Esquema da aproximação da corrente no ponto $x_{n-1}$ para o nó $n$.

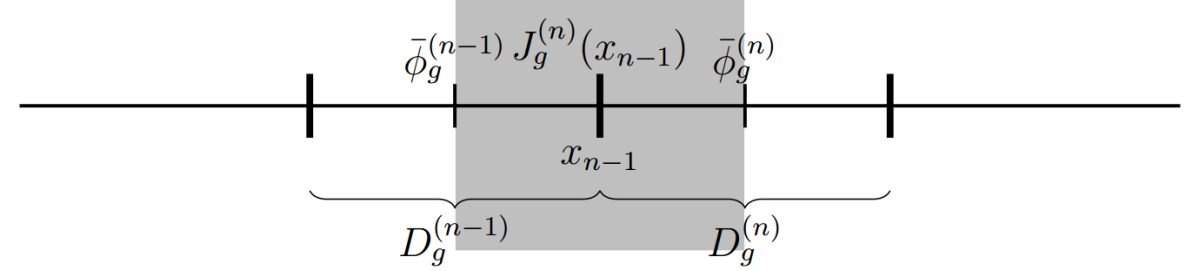

Fonte: Elaboração dos autores.

Assim, a aproximação da densidade de corrente para a interface, à direita do nó $n$, é expressa como

$$
J_{g}^{(n)}\left(x_{n}\right) \cong-\frac{D_{g}^{(n+1)}+D_{g}^{(n)}}{2}\left(\frac{\bar{\phi}_{g}^{(n+1)}-\bar{\phi}_{g}^{(n)}}{\Delta x}\right)
$$

e a aproximação para a interface, à esquerda do nó $n$, é expressa como

$$
J_{g}^{(n)}\left(x_{n-1}\right) \cong-\frac{D_{g}^{(n)}+D_{g}^{(n-1)}}{2}\left(\frac{\bar{\phi}_{g}^{(n)}-\bar{\phi}_{g}^{(n-1)}}{\Delta x}\right)
$$

Nas quatro propostas de aproximação das densidades de corrente, temos o mesmo esquema de aproximação da derivada. Dessa forma, a fim de buscarmos uma generalização, definimos como $\alpha_{g}^{(n)}$ os coeficientes que acompanham a aproximação da derivada na face direita e $\beta_{g}^{(n)}$ na face esquerda para cada nó $n$ e grupo $g$. Substituindo estas aproximações na Eq. (6), obtemos depois de algumas manipulações, 


$$
-\frac{\alpha_{g}^{(n)}}{\Delta x^{2}} \bar{\phi}_{g}^{(n+1)}+\left(\frac{\alpha_{g}^{(n)}+\beta_{g}^{(n)}}{\Delta x^{2}}\right) \bar{\phi}_{g}^{(n)}-\frac{\beta_{g}^{(n)}}{\Delta x^{2}} \bar{\phi}_{g}^{(n-1)}+\Sigma_{R g}^{(n)} \bar{\phi}_{g}^{(n)}=\frac{\chi_{g}}{K} \sum_{g^{\prime}=1}^{G} \nu_{g^{\prime}} \Sigma_{f g^{\prime}}^{(n)} \bar{\phi}_{g^{\prime}}^{(n)}+\sum_{\substack{g^{\prime}=1 \\ g^{\prime} \neq g}}^{G} \Sigma_{s g^{\prime} g}^{(n)} \bar{\phi}_{g^{\prime}}^{(n)}
$$

A Eq. 20 refere-se aos nós internos do domínio. Entretanto, as equações referentes aos nós de ambas extremidades do domínio são um pouco diferentes, pois sofrem influência das condições de contorno, Eq. (2). Estas equações são construídas a partir das seguintes aproximações das densidades de corrente para os contornos, que são iguais para as 4 propostas,

$$
J_{g}^{(1)}\left(x_{0}\right) \cong-\left(\frac{2 D_{g}^{(1)} a_{g}}{a_{g} \Delta x-2 b_{g}}\right) \bar{\phi}_{g}^{(1)}
$$

e

$$
J_{g}^{(N)}\left(x_{N}\right) \cong\left(\frac{2 D_{g}^{(N)} a_{g}}{a_{g} \Delta x+2 b_{g}}\right) \bar{\phi}_{g}^{(N)}
$$

O conjunto de todas as $G N$ equações obtidas formam um problema de autovalor que pode ser resolvido por diversas abordagens, entretanto, neste trabalho, destacamos duas delas. A primeira abordagem se baseia na avaliação das raízes de uma equação característica, com duas versões, dadas pelo método da bissecção e o método da secante. Uma segunda abordagem é a do método iterativo de fonte.

\subsection{Métodos da bissecção e da secante}

A partir das raízes do determinante da matriz de um sistema homogêneo, buscamos os valores de $K$ em que tal determinante seja igual a zero. Esse sistema homogêneo pode ser escrito como

$$
\mathbf{A} \bar{\Phi}=0
$$

onde $\bar{\Phi}$ é o vetor de ordem $G N$ cujos elementos são os fluxos médios em cada grupo e nó

$$
\overline{\mathbf{\Phi}}=\left[\bar{\phi}_{1}^{(1)}, \ldots, \bar{\phi}_{G}^{(1)}, \ldots, \bar{\phi}_{1}^{(N)}, \ldots, \bar{\phi}_{G}^{(N)}\right]^{T}
$$


e A é uma matriz tridiagonal de blocos de ordem $G N \times G N$,

$$
\mathbf{A}=\left[\begin{array}{ccccc}
\mathbf{A}_{q}^{(1)} & \mathbf{A}_{\alpha}^{(1)} & & & \\
\mathbf{A}_{\beta}^{(2)} & \mathbf{A}_{q}^{(2)} & \mathbf{A}_{\alpha}^{(2)} & & \\
& \ddots & \ddots & \ddots & \\
& & \mathbf{A}_{\beta}^{(N-1)} & \mathbf{A}_{q}^{(N-1)} & \mathbf{A}_{\alpha}^{(N-1)} \\
& & & \mathbf{A}_{\beta}^{(N)} & \mathbf{A}_{q}^{(N)}
\end{array}\right]
$$

onde os blocos $\mathbf{A}_{\beta}^{(n)}$ e $\mathbf{A}_{\alpha}^{(n)}$ de ordem $G \times G$ são matrizes diagonais da forma

$$
\mathbf{A}_{\beta}^{(n)}=\operatorname{diag}\left(-\frac{\beta_{1}^{(n)}}{\Delta x^{2}}, \ldots,-\frac{\beta_{G}^{(n)}}{\Delta x^{2}}\right)
$$

e

$$
\mathbf{A}_{\alpha}^{(n)}=\operatorname{diag}\left(-\frac{\alpha_{1}^{(n)}}{\Delta x^{2}}, \ldots,-\frac{\alpha_{G}^{(n)}}{\Delta x^{2}}\right)
$$

e os blocos da diagonal principal $\mathbf{A}_{q}^{(n)}$ de ordem $G \times G$ são matrizes cheias cujos elementos são definidos como

$$
\mathbf{A}_{q i, j}^{(n)}=\left\{\begin{array}{cl}
\left(\frac{\alpha_{i}^{(n)}+\beta_{i}^{(n)}}{\Delta x^{2}}\right)+\Sigma_{R i}^{(n)}-\frac{\chi_{i}}{K} \nu_{j} \Sigma_{f j}^{(n)} & \text { se } i=j \\
-\frac{\chi_{i}}{K} \nu_{j} \Sigma_{f j}^{(n)}-\Sigma_{s j i}^{(n)} & \text { se } i \neq j
\end{array} .\right.
$$

Para esse tipo de abordagem, propomos dois métodos para determinação das raízes da equação característica: método da bissecção e método da secante (EHIWARIO; AGHAMIE, 2014). Para isso, definimos o determinante da matriz $A$ como uma função $f$ que depende de $K$ da forma $\operatorname{det}(A(K))=f(K)$. Estes dois métodos partem de duas estimativas iniciais, $K^{[1]}$ e $K^{[2]}$, e com sucessivas iterações convergem para uma raiz da função $f$. As fórmulas de iteração são definidas como (EHIWARIO; AGHAMIE, 2014)

- Método da bissecção

$$
K^{[s+1]}=\frac{K^{[s-1]}+K^{[s]}}{2} .
$$

- Método da secante 


$$
K^{[s+1]}=\frac{K^{[s-1]} f\left(K^{[s]}\right)-K^{[s]} f\left(K^{[s-1]}\right)}{f\left(K^{[s]}\right)-f\left(K^{[s-1]}\right)} .
$$

Em ambos os casos, o processo iterativo deve seguir até que um critério de parada seja atingido, como por exemplo,

$$
\frac{\left|K^{[s+1]}-K^{[s]}\right|}{\left|K^{[s+1]}\right|}<\epsilon_{1}
$$

ou que um número limite de iterações seja alcançado (em caso de não convergência).

Em geral, o método da secante é formalmente o mais eficaz entre estes dois métodos (EHIWARIO; AGHAMIE, 2014). Entretanto, segundo Datta (2009), o método da bissecção sempre converge, em contraposição com o método da secante, que converge sob certas condições, por exemplo, $f\left(K^{[s]}\right)$ e $f\left(K^{[s-1]}\right)$ não podem ser suficientemente próximos. Além disso, esses métodos convergem para um autovalor (raiz), não necessariamente o maior deles. Como nosso problema de interesse requer apenas o autovalor dominante, precisamos elaborar algumas estratégias para determiná-lo. Visto que o método da secante não requer que as estimativas iniciais intercalem algum autovalor, propomos que as duas estimativas iniciais sejam um pouco maiores do que 1 , uma vez que o valor de $K$ crítico dos reatores nucleares são valores próximos de 1. Com estas estimativas, o método da secante converge na direção do autovalor dominante.

Após encontrarmos o autovalor $K$ pelas duas abordagens, podemos determinar os fluxos. Para isso, além de substituir $K$ no sistema homogêneo, Eq. (23), acrescentamos mais uma linha no sistema, que se refere ao cálculo da potência do reator

$$
P=E \sum_{n=1}^{N} \sum_{g=1}^{G} \nu_{g} \Sigma_{f g}^{(n)} \bar{\phi}_{g}^{(n)} \Delta x
$$

onde $E$ é a quantidade de energia que em média é gerada por fissão. Assim, a partir de uma potência prescrita, o sistema torna-se sobredeterminado e não homogêneo. Este novo sistema pode ser resolvido por mínimos quadrados. 


\subsection{Método iterativo de fonte}

A segunda abordagem que propomos é o método iterativo de fonte, que determina o autovalor dominante e seu autovetor correspondente, através de sucessivas iterações (DUDERSTADT; HAMILTON, 1976). Nesta abordagem organizamos as equações do problema da seguinte forma

$$
\mathbf{M} \overline{\mathbf{\Phi}}=\frac{1}{K} \mathbf{F} \overline{\mathbf{\Phi}}
$$

onde $\mathbf{M}$ é uma matriz tridiagonal de blocos semelhante à matriz $\mathbf{A}$ da $\mathbf{E q}$. (25). A diferença está apenas nos blocos da diagonal principal, pois os elementos $\frac{\chi_{i}}{K} \nu_{j} \Sigma_{f j}^{(n)}$ não fazem parte da matriz $\mathbf{M}$. Nesse caso, as seções de choque de fissão estão no lado direito do sistema matricial, formando a matriz $\mathbf{F}$ de ordem $G N \times G N$, que é uma matriz diagonal de blocos da forma

$$
\mathbf{F}=\operatorname{diag}\left(\mathbf{F}_{d}^{(1)}, \ldots, \mathbf{F}_{d}^{(N)}\right)
$$

onde os blocos $\mathbf{F}_{d}^{(n)}$ de ordem $G \times G$ são matrizes da forma

$$
\mathbf{F}_{d}^{(n)}=\left[\begin{array}{ccc}
f_{1,1}^{(n)} & \cdots & f_{1, G}^{(n)} \\
\vdots & \ddots & \vdots \\
f_{G, 1}^{(n)} & \cdots & f_{G, G}^{(n)}
\end{array}\right]
$$

para $f_{i, j}^{(n)}=\chi_{i} \nu_{j} \Sigma_{f j}^{(n)}$. A ideia principal desse método é assumir que o lado direito (termo fonte) da Eq. (33) seja sempre conhecido, na forma de um processo iterativo,

$$
\mathbf{M} \overline{\mathbf{\Phi}}^{[s]}=\frac{1}{K^{[s-1]}} \mathbf{F} \overline{\mathbf{\Phi}}^{[s-1]} .
$$

Iniciamos o processo com estimativas iniciais para o $K^{[0]}$ e $\overline{\mathbf{\Phi}}^{[0]}$. Com o lado direito conhecido, obtemos um sistema linear que, quando resolvido, determina a atualização dos fluxos e subsequente atualização do $K$. A fórmula de atualização do $K$ segue a proposta de Duderstadt e Hamilton (1976), e é escrita na forma

$$
K^{[s]}=K^{[s-1]} \frac{\left\langle\mathbf{F} \overline{\boldsymbol{\Phi}}^{[s]}, \mathbf{F} \overline{\mathbf{\Phi}}^{[s]}\right\rangle}{\left\langle\mathbf{F} \overline{\boldsymbol{\Phi}}^{[s-1]}, \mathbf{F} \overline{\boldsymbol{\Phi}}^{[s]}\right\rangle},
$$


onde o símbolo $\langle\boldsymbol{u}, \boldsymbol{v}\rangle$ significa o produto interno. Esse método deve iterar até atender algum critério de parada, como

$$
\frac{\left\|\overline{\boldsymbol{\Phi}}^{[s]}-\overline{\boldsymbol{\Phi}}^{[s-1]}\right\|_{2}}{\left\|\overline{\boldsymbol{\Phi}}^{[s]}\right\|_{2}}<\epsilon_{2},
$$

além do critério apresentado na Eq. (31). Esse método iterativo de fonte determina o autovalor dominante, $K$, independentemente da estimativa inicial, desde que exista uma solução (DUDERSTADT; HAMILTON, 1976).

\section{Resultados numéricos}

A fim de analisarmos as propostas de aproximação das densidades de correntes e os métodos para determinação da criticalidade, resolvemos três problemas testes presentes na literatura (SILVA; MARTINEZ; GONÇALVES, 2012, THOMÉ; CARVALHO; ALVIM, 1997, POLLARD, 1977). Os resultados numéricos para os problemas testes são gerados por códigos implementados em Fortran95 e executados em um computador pessoal com um processador Intel Core i5-8250U, de 1,60GHz e 8GB de RAM. Os tempos computacionais apresentados nas tabelas a seguir são determinados a partir da média aritmética de 10 execuções do código. Além disso, as estimativas iniciais para o método da secante dos três problemas testes são $K^{[1]}=1,3$ e $K^{[2]}=1,2$.

\subsection{Problema Teste 1}

O primeiro problema teste foi proposto por Silva, Martinez e Gonçalves (2012). Consideramos um meio heterogêneo com dois tipos de materiais: um combustível, $0 \leq x \leq 30(\mathrm{~cm})$, e outro refletor $30 \leq x \leq 50(\mathrm{~cm})$. As condições de contorno impostas são do tipo reflexivas no contorno esquerdo e fluxos nulos no contorno direito, respectivamente,

$$
\left.\frac{d}{d x} \phi_{g}(x)\right|_{x=0}=0,\left.\quad \phi_{g}(x)\right|_{x=50}=0
$$

Os critérios de parada que adotamos são $\epsilon_{1}=10^{-10}$ e $\epsilon_{2}=10^{-10}$. Na Tabela 1 , apresentamos os parâmetros nucleares do problema. 
Tabela 1 - Parâmetros nucleares para o Problema Teste 1.

\begin{tabular}{ccccccc}
\hline Material & $\boldsymbol{g}$ & $\boldsymbol{D}_{\boldsymbol{g}}(\mathrm{cm})$ & $\boldsymbol{\Sigma}_{\boldsymbol{R} \boldsymbol{g}}\left(\mathrm{cm}^{-1}\right)$ & $\nu_{\boldsymbol{g}} \boldsymbol{\Sigma}_{\boldsymbol{f g}}\left(\mathrm{cm}^{-1}\right)$ & $\boldsymbol{\Sigma}_{\boldsymbol{s g} \mathbf{2}}\left(\mathrm{cm}^{-1}\right)$ & $\chi_{\boldsymbol{g}}$ \\
\hline 1 & 1 & 1,438000 & 0,029350 & 0,000242 & 0,01563 & 1,0 \\
1 & 2 & 0,397600 & 0,104900 & 0,155618 & 0,00000 & 0,0 \\
2 & 1 & 1,871420 & 0,035411 & 0,000000 & 0,03434 & 1,0 \\
2 & 2 & 0,283409 & 0,031579 & 0,000000 & 0,00000 & 0,0 \\
\hline
\end{tabular}

Fonte: Adaptado de Silva, Martinez e Gonçalves (2012, p. 185).

Na Tabela 2, apresentamos os resultados encontrados para o autovalor dominante $K$, utilizando as quatro propostas para diferentes $\Delta x$ e seus tempos de execução na coluna subsequente. As estimativas iniciais do método da bissecção são $K^{[1]}=1,0$ e $K^{[2]}=0,6$ e o resultado do $K$, apresentado por Silva, Martinez e Gonçalves (2012), é de 0,7346988.

Tabela 2 - Resultados para o autovalor dominante $K$ para o Problema Teste 1.

\begin{tabular}{|c|c|c|c|c|c|c|c|}
\hline Método & Proposta & $\Delta x=1 \mathrm{~cm}$ & $t(s)$ & $\Delta x=0,5 \mathrm{~cm}$ & $t(s)$ & $\Delta x=0,25 \mathrm{~cm}$ & $t(s)$ \\
\hline \multirow{4}{*}{ Bis. } & 1 & 0,74923 & 0,010 & 0,74970 & 0,027 & 0,74982 & 0,071 \\
\hline & 2 & 0,73548 & 0,010 & 0,73526 & 0,023 & 0,73510 & 0,078 \\
\hline & 3 & 0,73470 & 0,011 & 0,73486 & 0,023 & 0,73490 & 0,067 \\
\hline & 4 & 0,73471 & 0,010 & 0,73487 & 0,024 & 0,73491 & 0,069 \\
\hline \multirow{4}{*}{ Sec. } & 1 & 0,74923 & 0,004 & 0,74970 & 0,007 & 0,74982 & 0,016 \\
\hline & 2 & 0,73548 & 0,004 & 0,73526 & 0,007 & 0,73510 & 0,016 \\
\hline & 3 & 0,73470 & 0,004 & 0,73486 & 0,007 & 0,73490 & 0,018 \\
\hline & 4 & 0,73471 & 0,004 & 0,73487 & 0,007 & 0,73491 & 0,016 \\
\hline \multirow{4}{*}{ I.F. } & 1 & 0,74923 & 0,006 & 0,74970 & 0,023 & 0,74982 & 0,091 \\
\hline & 2 & 0,73548 & 0,007 & 0,73526 & 0,021 & 0,73510 & 0,076 \\
\hline & 3 & 0,73470 & 0,007 & 0,73486 & 0,023 & 0,73490 & 0,081 \\
\hline & 4 & 0,73471 & 0,007 & 0,73487 & 0,021 & 0,73491 & 0,081 \\
\hline
\end{tabular}

Fonte: Dados da pesquisa.

A principal informação que podemos tirar deste problema teste, Tabela 2, é a discrepância dos resultados da Proposta 1, essa característica se repete nos demais problemas teste. Isso ocorre porque há uma descontinuidade nas interfaces devido à aproximação da densidade de corrente, conforme já mencionado na formulação do problema. Além disso, podemos perceber que os três métodos testados obtiveram pelo menos 5 dígitos de concordância, quando comparamos as mesmas propostas de aproximação das correntes. Entretanto, o método da secante demanda menos 
tempo computacional. Ademais, os resultados obtidos concordam em até 3 dígitos significativos com o trabalho apresentado por Silva, Martinez e Gonçalves (2012), que utilizam o método das diferenças finitas, porém eles não apresentam maiores detalhes como, por exemplo, tamanho da malha utilizada.

\subsection{Problema Teste 2}

Este segundo problema teste foi proposto por Thomé, Carvalho e Alvim (1997), no qual consideramos um meio heterogêneo com três tipos de materiais dispostos em uma placa de $105 \mathrm{~cm}$, com cinco regiões. As condições de contorno impostas são do tipo fluxos nulos em ambos os contornos,

$$
\left.\phi_{g}(x)\right|_{x=0}=0,\left.\quad \phi_{g}(x)\right|_{x=105}=0
$$

Os critérios de parada que utilizamos são $\epsilon_{1}=10^{-10}$ e $\epsilon_{2}=10^{-8}$. Na Tabela 3 , apresentamos os parâmetros nucleares para os três tipos de materiais.

Tabela 3 - Parâmetros nucleares para o Problema Teste 2.

\begin{tabular}{ccccccc}
\hline Material & $g$ & $D_{g}(\mathrm{~cm})$ & $\Sigma_{R g}\left(\mathrm{~cm}^{-1}\right)$ & $\nu_{g} \Sigma_{f g}\left(\mathrm{~cm}^{-1}\right)$ & $\Sigma_{s g 2}\left(\mathrm{~cm}^{-1}\right)$ & $\chi_{g}$ \\
\hline 1 & 1 & 1,6562 & 0,02299 & 0,005607 & 0,01391 & 1,0 \\
1 & 2 & 0,4749 & 0,07637 & 0,116780 & 0,00000 & 0,0 \\
2 & 1 & 1,6617 & 0,02293 & 0,006182 & 0,01363 & 1,0 \\
2 & 2 & 0,4740 & 0,08547 & 0,135300 & 0,00000 & 0,0 \\
3 & 1 & 0,6702 & 0,09213 & 0,000000 & 0,09084 & 1,0 \\
3 & 2 & 0,1509 & 0,02072 & 0,000000 & 0,00000 & 0,0 \\
\hline
\end{tabular}

Fonte: Adaptado de Thomé, Carvalho e Alvim (1997, p. 959).

$\mathrm{Na}$ Tabela 4, apresentamos os resultados encontrados para o $K$, utilizando diferentes $\Delta x \mathrm{e}$ seus respectivos tempos de execução, na coluna subsequente. As estimativas iniciais do método da bissecção são $K^{[1]}=1,2$ e $K^{[2]}=0,95$ e o autovalor $K$ apresentado por Thomé, Carvalho e Alvim (1997) é 1,1051. 
Tabela 4 - Resultados para o autovalor dominante $K$ para o Problema Teste 2.

\begin{tabular}{|c|c|c|c|c|c|c|c|}
\hline Método & Proposta & $\Delta x=1 \mathrm{~cm}$ & $t(s)$ & $\Delta x=0,5 \mathrm{~cm}$ & $t(s)$ & $\Delta x=0,25 \mathrm{~cm}$ & $t(s)$ \\
\hline \multirow{4}{*}{ Bis. } & 1 & 1,07135 & 0,024 & 1,07184 & 0,075 & 1,07197 & 0,480 \\
\hline & 2 & 1,10364 & 0,025 & 1,10398 & 0,076 & 1,10406 & 0,589 \\
\hline & 3 & 1,10312 & 0,023 & 1,10383 & 0,075 & 1,10402 & 0,469 \\
\hline & 4 & 1,10398 & 0,023 & 1,10429 & 0,073 & 1,10425 & 0,445 \\
\hline \multirow{4}{*}{ Sec. } & 1 & 1,07135 & 0,015 & 1,07184 & 0,024 & 1,07197 & 0,072 \\
\hline & 2 & 1,10364 & 0,011 & 1,10398 & 0,028 & 1,10406 & 0,073 \\
\hline & 3 & 1,10312 & 0,010 & 1,10383 & 0,021 & 1,10402 & 0,071 \\
\hline & 4 & 1,10398 & 0,009 & 1,10429 & 0,028 & 1,10425 & 0,070 \\
\hline \multirow{4}{*}{ I.F. } & 1 & 1,07135 & 0,027 & 1,07184 & 0,088 & 1,07197 & 0,272 \\
\hline & 2 & 1,10364 & 0,044 & 1,10398 & 0,154 & 1,10406 & 0,413 \\
\hline & 3 & 1,10312 & 0,025 & 1,10383 & 0,093 & 1,10402 & 0,270 \\
\hline & 4 & 1,10398 & 0,025 & 1,10429 & 0,089 & 1,10425 & 0,278 \\
\hline
\end{tabular}

Fonte: Dados da pesquisa.

Além da discordância dos resultados da Proposta 1, na Tabela 4, podemos notar que a Proposta 2 demanda um maior custo computacional em comparação com as demais propostas. Além disso, podemos perceber novamente que os três métodos propostos obtiveram os mesmos 6 primeiros dígitos e que o método da secante demanda menos tempo computacional. Ademais, os resultados obtidos nas Propostas 2, 3 e 4 concordam em até 3 dígitos significativos com o trabalho apresentado por Thomé, Carvalho e Alvim (1997).

\subsection{Problema Teste 3}

Este é um problema clássico na literatura e é nomeado Benchmark BSS-6 (POLLARD, 1977). Consideramos um meio heterogêneo, com dois tipos de materiais, dispostos em três regiões, em uma placa de $240 \mathrm{~cm}$, na qual as regiões das extremidades são de mesmo material (material 1 da Tabela 5. As condições de contorno impostas para esse problema são de fluxos nulos em ambos os contornos,

$$
\left.\phi_{g}(x)\right|_{x=0}=0,\left.\quad \phi_{g}(x)\right|_{x=240}=0
$$


Os critérios de parada que utilizamos são $\epsilon_{1}=10^{-8}$ e $\epsilon_{2}=10^{-6}$. Na Tabela 5 , apresentamos os parâmetros nucleares para os dois tipos de materiais.

Tabela 5 - Parâmetros nucleares para o Problema Teste 3.

\begin{tabular}{ccccccc}
\hline Material & $\boldsymbol{g}$ & $\boldsymbol{D}_{\boldsymbol{g}}(\mathbf{c m})$ & $\boldsymbol{\Sigma}_{\boldsymbol{R g}}\left(\mathrm{cm}^{-1}\right)$ & $\boldsymbol{\nu}_{\boldsymbol{g}} \boldsymbol{\Sigma}_{\boldsymbol{f g}}\left(\mathrm{cm}^{-1}\right)$ & $\Sigma_{\boldsymbol{s g} \mathbf{2}}\left(\mathrm{cm}^{-1}\right)$ & $\chi_{\boldsymbol{g}}$ \\
\hline 1 & 1 & 1,5 & 0,026 & 0,010 & 0,015 & 1,0 \\
1 & 2 & 0,5 & 0,180 & 0,200 & 0,000 & 0,0 \\
2 & 1 & 1,0 & 0,020 & 0,005 & 0,010 & 1,0 \\
2 & 2 & 0,5 & 0,080 & 0,099 & 0,000 & 0,0 \\
\hline
\end{tabular}

Fonte: Adaptado de Pollard (1977, p. 7).

Na Tabela 6, apresentamos os resultados encontrados para o autovalor dominante $K$, utilizando diferentes $\Delta x$ e seus tempos de execução, na coluna subsequente. As estimativas iniciais do método da bissecção são $K^{[1]}=1,1$ e $K^{[2]}=0,901$ e o autovalor dominante $K$ apresentado por Pollard (1977) é 0,90156 .

Tabela 6 - Resultados para o autovalor dominante $K$ para o Problema Teste 3.

\begin{tabular}{|c|c|c|c|c|c|c|c|}
\hline Método & Proposta & $\Delta x=1 \mathrm{~cm}$ & $t(s)$ & $\Delta x=0,5 \mathrm{~cm}$ & $t(s)$ & $\Delta x=0,25 \mathrm{~cm}$ & $t(s)$ \\
\hline \multirow{4}{*}{ Bis. } & 1 & - & - & - & - & - & - \\
\hline & 2 & 0,901642 & 0,071 & 0,901608 & 0,272 & 0,901599 & 1,799 \\
\hline & 3 & 0,901632 & 0,072 & 0,901606 & 0,271 & 0,901599 & 1,837 \\
\hline & 4 & 0,901610 & 0,078 & 0,901594 & 0,264 & 0,901593 & 1,811 \\
\hline \multirow{4}{*}{ Sec. } & 1 & 0,892037 & 0,078 & 0,891973 & 0,275 & 0,891956 & 1,888 \\
\hline & 2 & 0,901642 & 0,075 & 0,901608 & 0,268 & 0,901599 & 2,007 \\
\hline & 3 & 0,901632 & 0,076 & 0,901606 & 0,287 & 0,901599 & 2,120 \\
\hline & 4 & 0,901610 & 0,076 & 0,901594 & 0,286 & 0,901593 & 2,099 \\
\hline \multirow{4}{*}{ I.F. } & 1 & 0,892038 & 0,310 & 0,891973 & 1,119 & 0,891956 & 4,455 \\
\hline & $2^{*}$ & 0,901642 & 2,584 & 0,901608 & 7,995 & 0,901599 & 21,069 \\
\hline & 3 & 0,901632 & 0,276 & 0,901606 & 0,978 & 0,901599 & 3,769 \\
\hline & 4 & 0,901610 & 0,277 & 0,901595 & 0,977 & 0,901593 & 3,776 \\
\hline
\end{tabular}

Fonte: Dados da pesquisa.

Na Tabela 6, a linha referente ao método da bissecção e Proposta 1 não apresenta resultados, pois as estimativas iniciais para este problema não intercalam nenhum autovalor. Essa situação ocorre pois a Proposta 1 diverge das demais propostas, como podemos ver nos resultados dos demais métodos. Além disso, queremos observar que o sinal, *, na Proposta 2 do método iterativo de 
fonte, simboliza a necessidade de reduzir $\epsilon_{2}$ para $10^{-5}$, a fim de que este código convirja em até 2000 iterações. Esse fato sugere uma desvantagem, principalmente quando queremos os valores precisos dos fluxos. Outra informação importante a ser destacada é que o método da bissecção foi mais rápido do que o método da secante, pois a estimativa inicial $K=0,901$, do método da bissecção, foi muito próximo do autovalor dominante. Essa situação ocorreu, pois o segundo maior autovalor ( $K=0,900196)$ é muito próximo do autovalor dominante, assim, se tivéssemos adotado um intervalo maior para as estimativas iniciais, o método poderia convergir para outro autovalor ou não atenderia a hipótese, $f\left(K^{[1]}\right) \cdot f\left(K^{[2]}\right)<0$. Por esses motivos, algumas dificuldades podem ser encontradas ao aplicar o método para problemas de autovalor dominante.

Ainda na Tabela 6, podemos observar que os três métodos obtiveram, pelo menos, 5 dígitos de concordância, quando comparadas as mesmas propostas de aproximação das densidades de corrente. Ademais, os resultados obtidos nas Propostas 2, 3 e 4 concordam em até 4 dígitos com a referência adotada, o que sugere uma boa concordância para cálculos globais em física de reatores.

Além do autovalor dominante $K$, neste problema teste de referência, queremos também comparar os resultados das frações de potência e dos fluxos escalares de nêutrons. As frações de potência, ou seja, os percentuais de potência gerados pelas três regiões materiais do problema, apresentadas por Pollard (1977) são 0,2790, para a primeira e a terceira região, e 0,4421, para a segunda região. Na Tabela 7, apresentamos as frações de potência encontradas pelas metodologias propostas neste trabalho, quando utilizamos $\Delta x=0,25 \mathrm{~cm}$, e o erro relativo percentual.

Tabela 7 - Resultados para as frações de potência por região.

\begin{tabular}{cccccc}
\hline Método & Região & Proposta 1 & Proposta 2 & Proposta 3 & Proposta 4 \\
\hline \multirow{2}{*}{ Bis. } & 1 & - & $0,27267(2,27 \%)$ & $0,27886(0,05 \%)$ & $0,27882(0,06 \%)$ \\
& 2 & - & $0,44228(0,04 \%)$ & $0,44229(0,04 \%)$ & $0,44236(0,06 \%)$ \\
& 3 & - & $0,28505(2,17 \%)$ & $0,27886(0,05 \%)$ & $0,27882(0,06 \%)$ \\
\hline \multirow{2}{*}{ Sec. } & 1 & $0,26731(4,19 \%)$ & $0,27267(2,27 \%)$ & $0,27886(0,05 \%)$ & $0,27882(0,06 \%)$ \\
& 2 & $0,46538(5,27 \%)$ & $0,44228(0,04 \%)$ & $0,44229(0,04 \%)$ & $0,44236(0,06 \%)$ \\
& 3 & $0,26731(4,19 \%)$ & $0,28505(2,17 \%)$ & $0,27886(0,05 \%)$ & $0,27882(0,06 \%)$ \\
I.F. & 1 & $0,26731(4,19 \%)$ & $0,27439(1,65 \%)$ & $0,27885(0,05 \%)$ & $0,27882(0,06 \%)$ \\
& 2 & $0,46539(5,27 \%)$ & $0,44228(0,04 \%)$ & $0,44230(0,05 \%)$ & $0,44237(0,06 \%)$ \\
& 3 & $0,26731(4,19 \%)$ & $0,28333(1,55 \%)$ & $0,27885(0,05 \%)$ & $0,27882(0,06 \%)$ \\
\hline
\end{tabular}

Fonte: Dados da pesquisa. 
O primeiro aspecto que queremos destacar dos resultados presentes, na Tabela 7 , é a não simetria das frações de potência obtidos pela Proposta 2. Como o problema é simétrico, os fluxos escalares também devem ser, assim como as potências. Esse fato indica uma certa deficiência da Proposta 2, na aproximação das densidades de correntes. Além disso, podemos observar que os resultados das Propostas 3 e 4 possuem boa concordância entre si e com a referência adotada. $\mathrm{Na}$ Figura 2, apresentamos os gráficos dos fluxos escalares de nêutrons, as linhas de maior amplitude são do grupo 1 (rápido) e as de menor amplitude são do grupo 2 (térmico). O gráfico (a) apresenta os fluxos das 4 propostas para o método da secante e o gráfico (b) apresenta os fluxos das 4 propostas para os métodos da secante e iterativo de fonte.

Figura 2 - Fluxos escalares de nêutrons rápido e térmico.

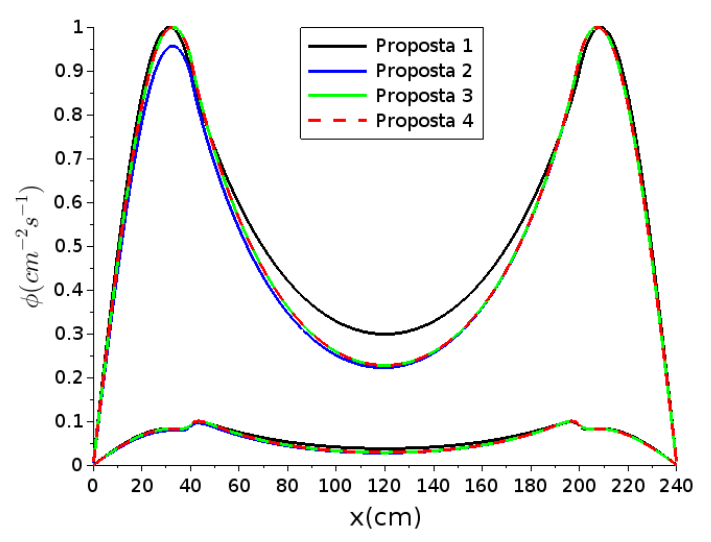

Gráfico (a)

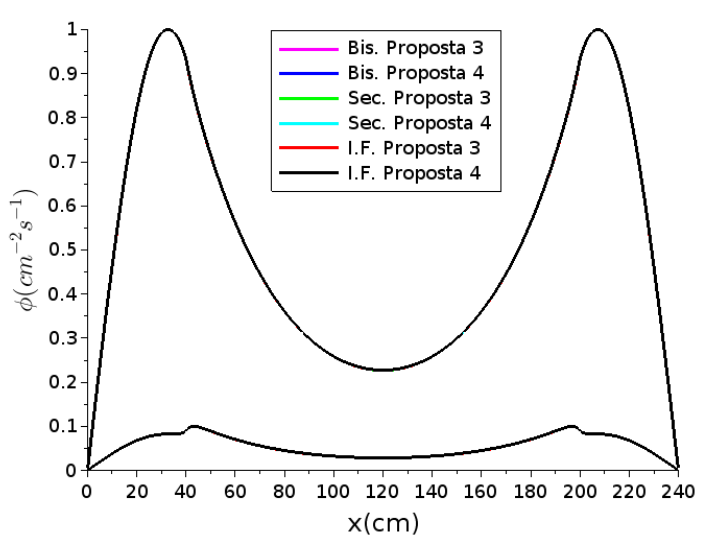

Gráfico (b)

Fonte: Elaboração dos autores.

No gráfico (a) da Figura 2, podemos observar a discrepância entre os fluxos das Propostas 1 e 2 em relação às Propostas 3 e 4 . Essa observação confirma o que já havíamos constatado nos resultados apresentados anteriormente. Além disso, nos dois gráficos, podemos perceber que os fluxos das Propostas 3 e 4 são muito próximos, tanto que em ambas as figuras a diferença é impercetível. Por fim, essa pequena diferença entre os fluxos das Propostas 3 e 4 pode ser observada na Tabela 8 , onde apresentamos o máximo valor absoluto das componentes do vetor diferença entre os fluxos escalares médios dos diferentes métodos e propostas. 
Tabela 8 - Comparação entre fluxos escalares de nêutrons.

\begin{tabular}{l|l}
\hline $\max \left(\left|\bar{\Phi}_{\text {Sec.Prop. } 4}-\bar{\Phi}_{\text {Bis.Prop.4 }}\right|\right)=1,5 \times 10^{-8}$ & $\max \left(\left|\bar{\Phi}_{\text {Sec.Prop. } 3}-\bar{\Phi}_{\text {Bis.Prop. } 3}\right|\right)=8,6 \times 10^{-9}$ \\
\hline $\max \left(\left|\bar{\Phi}_{\text {Sec.Prop. } 4}-\bar{\Phi}_{\text {I.F.Prop. } 4}\right|\right)=2,4 \times 10^{-5}$ & $\max \left(\left|\bar{\Phi}_{\text {Sec.Prop. } 3}-\bar{\Phi}_{\text {I.F.Prop. } 3}\right|\right)=2,4 \times 10^{-5}$ \\
\hline $\max \left(\left|\bar{\Phi}_{\text {Bis.Prop.4 }}-\bar{\Phi}_{\text {I.F.Prop. } 4}\right|\right)=2,4 \times 10^{-5}$ & $\max \left(\left|\bar{\Phi}_{\text {Bis.Prop. } 3}-\bar{\Phi}_{\text {I.F.Prop. } 3}\right|\right)=2,4 \times 10^{-5}$ \\
\hline $\max \left(\left|\bar{\Phi}_{\text {Sec.Prop. } 4}-\bar{\Phi}_{\text {Sec.Prop. } 3}\right|\right)=2,3 \times 10^{-4}$ & $\max \left(\left|\bar{\Phi}_{\text {Bis.Prop. } 4}-\bar{\Phi}_{\text {Bis.Prop. } 3}\right|\right)=2,3 \times 10^{-4}$ \\
\hline $\max \left(\left|\bar{\Phi}_{\text {I.F.Prop.4 }}-\bar{\Phi}_{\text {I.F.Prop. } 3}\right|\right)=2,3 \times 10^{-4}$ & \\
\hline
\end{tabular}

Fonte: Dados da pesquisa.

\section{Conclusões}

A teoria da difusão de nêutrons é amplamente utilizada na análise global de reatores nucleares modernos e, assim, estudos contínuos são necessários para buscar aprimoramentos nas atuais metodologias de solução. Neste trabalho, um dos objetivos foi analisar diferentes propostas da aproximação das densidade de corrente, principalmente para as interfaces dos nós, que definem uma malha computacional do domínio, uma vez que estes pontos são muito sensíveis em virtude da descontinuidade na composição material. Após os diversos testes avaliados, observamos que as Propostas 3 e 4 apresentaram um melhor desempenho, tanto na precisão como no tempo computacional.

Outro objetivo deste trabalho foi analisar diferentes métodos em cálculos de criticalidade (autovalor dominante) em reatores nucleares. Após os testes realizados, concluímos que o método da secante se mostrou mais eficiente do que os demais métodos propostos. Além disso, a estratégia de escolher estimativas iniciais um pouco maiores do que 1, para o método da secante, mostrou-se satisfatória, por convergir para o autovalor dominante. Para trabalhos futuros, pretendemos expandir este estudo comparativo para problemas multidimensionais.

\section{Agradecimentos}

Este estudo foi financiado em parte: pelo Conselho Nacional de Desenvolvimento Científico e Tecnológico (CNPq) - Brasil; pela Coordenação de Aperfeiçoamento de Pessoal de Nível Superior (CAPES) - Brasil: Código Financeiro 001; pela Fundação de Amparo à Pesquisa do Estado do Rio Grande do Sul (FAPERGS): Proc. 19/2551-0001766-9. 


\section{Referências}

ABREU, W. V. Análise numérica de transientes em um reator slab guiado por fonte externa. 2017. 95 f. Dissertação (Mestrado em Ciência e Tecnologia Nucleares) - Programa de Pós-Graduação em Ciência e Tecnologia Nucleares do Instituto de Engenharia Nuclear, Comissão Nacional de Engenharia Nuclear, Rio de Janeiro, 2017.

DATTA, B. N. Lecture notes on numerical solution of root finding problems. DeKalb: Northern Illinois University, 2009. Disponível em: http://www.math.niu.edu/ dattab/math435.2009/ROOT-FINDING.pdf. Acesso em: 20 jan. 2020.

DUDERSTADT, J. J; HAMILTON L. J. Nuclear reactor analysis. New York: John Wiley, 1976.

EHIWARIO, J. C; AGHAMIE, S. O. Comparative study of bisection, Newton-Raphson and secant methods of root - finding problems. IOSR Journal of Engineering, v. 4, n. 4, p. 1-7, abr. 2014. DOI: https://doi.org/10.9790/3021-04410107.

GROSSMAN, L. M; HENNART, J. P. Nodal diffusion methods for space-time neutron kinetics. Progress in Nuclear Energy, v. 49, n. 3, p. 181-216, 2007. DOI: https://doi.org/10.1016/j.pnucene.2006.11.003.

LAMARSH, J. R; BARATTA, A. J. Introduction to nuclear engineering. 4. ed. Pearson Education, 2017.

LEWIS, E. E. Fundamentals of nuclear reactor physics. Oxford: Elsevier's Science and Technology, 2008.

POLLARD, J. P. AUS diffusion module POW checkout - 1- and 2- dimensional kinetics calculations. Australian Atomic Energy Commission AAEC/387, 1977. Disponível em: https://inis.iaea.org/collection/NCLCollectionStore/_Public/08/328/8328678.pdf. Acesso em: 19 nov. 2020.

SILVA, A. C; MARTINEZ, A. S; GONÇALVES, A. da C. Reconstruction of the neutron flux in a slab reactor. World Journal of Nuclear Science and Technology, v. 2, p. 181-186, 2012. DOI: http://dx.doi.org/10.4236/wjnst.2012.24028.

STACEY, W. M. Nuclear reactor physics. New York: John Wiley \& Sons, 2001.

THOMÉ, Z; CARVALHO, F. da S; ALVIM, A. Application of local basis pseudo-harmonics method. Annals of Nuclear Energy, v. 24, n. 12, p. 955-963, 1997. DOI: https://doi.org/10.1016/S0306-4549(96)00090-4. 